\title{
Regulated Morphogen Gradients for Target Surrounding and Adaptive Shape Formation
}

\author{
Ataollah Ramezan Shirazi and Yaochu Jin, Fellow, IEEE
}

\begin{abstract}
In swarm robotics, developing algorithms for self-organizing minimalistic robots has become a popular research topic. Unlike others, minimalistic robots may not be able to self-localize themselves, making it very challenging to accomplish missions such as surrounding a target, whose position is typically unknown. In target surrounding, reaching a target and joining the swarm do not always lead to a satisfactory enclosure of the target. Furthermore, it is impossible for individual minimalistic robots to figure out what a global shape of the swarm should be without a collective decision-making. In this research, we make use of diffusion and reaction of two morphogens for target surrounding and formation of a circular shape swarm. We show that the proposed method is able to adaptively form shapes surrounding multiple targets. Computer simulations and physical experiments using Kilobots are performed to assess the performance of the proposed algorithm.
\end{abstract}

Index Terms-Swarm robotics, target enclosure, flocking, artificial morphogen, self-organising systems

\section{INTRODUCTION}

\section{A. Overview}

One main objective of swarm robotic research is to design algorithms for self-organization of a large number of highly restricted robots to accomplish a common task. Self-organized surrounding of diffusive targets with a swarm of simplistic robots is a challenging research topic having numerous potential applications, such as odour source localization [1], gas leak detection [2], and seeking and destroying cancer cells in medical nanorobotics [3]. Target surrounding is also a necessary step for producing other emerging collective behaviors such as collective transportation and shape formation.

Target enclosure usually starts with a large number of scattered and loosely connected robots (agents) that are foraging in an unknown environment. Target enclosure behaviors are widely observed in nature, including

Ataollah Ramezan Shirazi and Yaochu Jin are with the Department of Computer Science, University of Surrey, Guildford, Surrey, GU2 7XH, UK (email: \{a.ramezanshirazi, yaochu.jin\}@surrey.ac.uk).

This work was funded by the European Commission 7th Framework Program, Project no. 601062, SWARM-ORGAN. cellular systems and social animals. Target enclosure behaviors in cellular systems are particularly interesting because of the limited information the cells can gain and the limited mobility they have. This metaphor between the cellular systems and swarm robotics makes cellular behaviors a rich source of inspiration for designing swarm robotics.

One important property in target enclosure is the shape of the swarm around the targets, as this shape can facilitate future actions on the targets, such as group transportation, construction or destruction. Moreover, a disk-like shape around the targets can often result in robust enclosure. A circular swarm shape can also be an intermediate step for further actions requiring an equal number of robots to move in different directions. In this work, we show how the outer layer of a robotic swarm can govern the growth of an aggregation. Due to the lack of global information, it is impossible for the robots to directly evaluate the general form of the swarm around a target. Take the diffusion limited aggregation (DLA) model [4] as an example. In the model, randomly moving robots irreversibly join a swarm around a target (Fig. 1). Once an individual robot touches a swarm, it stops moving and becomes a part of swarm. As a result, a fractal-like tree structure rooted at the target seed can be formed. Examples of DLAs in nature include the formation of snowake and dust, and growth of coral. However, additional strategies are needed to control the global shape of the swarm based on local awareness of the individual robots.

This work addresses the problem of surrounding static targets using minimalist robots with limited omnidirectional sensing capability. Each target acts as a source of an artificial morphogen signal that is directly detectable only by the robots that are within the communication range of the target. Any robot receives this signal will pass it to its neighbors. This way, we can simulate morphogen diffusion that allows the robots to have a rough estimation of their distance to the target. The desired behavior is to enclose a target or targets evenly from all sides, which can be realized by forming a circular shape swarm. To this end, the robots need to be able to identify the positions on the edge of swarm where 


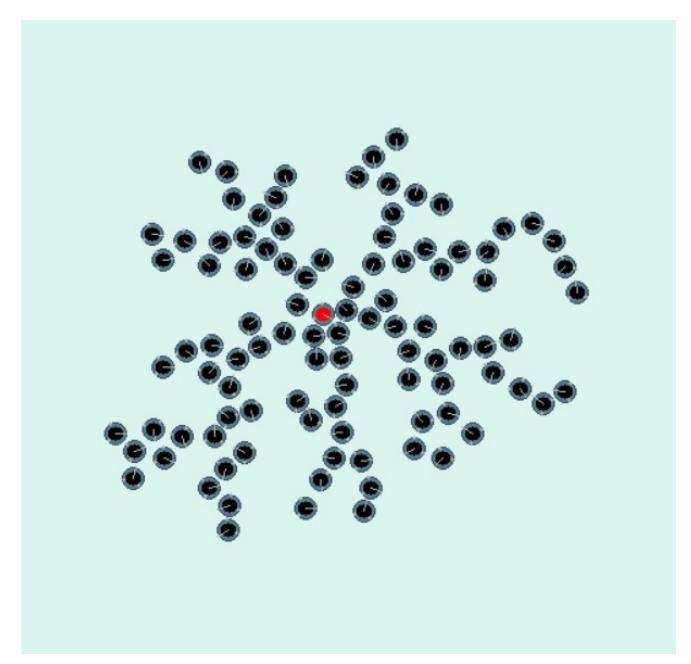

Fig. 1: Simulation of diffusion limited aggregation.

more robots should aggregate using a second morphogen regulated by the target morphogen.

\section{B. Background}

In the top-down approach to swarm robotic systems, each individual agent in the swarm is assumed to be able to estimate the states of the other agents within an appropriate time period before making a decision [5]. Such an approach is impractical for this work since it assumes that the global state of the swarm is accessible to all individual agents for planning their future actions. By contrast, the bottom-up approach is based on local awareness and local interactions of individual agents based on simple rules that are either manually designed or automatically evolved to achieve certain global behaviors [6]. For example, Brutschy et al. [7] designed a self-organizing algorithm for allocating collective complex tasks to a swarm of minimalist robots, where each complex task is divided into a sequence of simple subtasks. Then, the robots switch between subtasks to maximize their performance.

I is a common way of developing algorithms for swarm robotic systems [8] by getting inspirations from intelligent swarm behaviors in nature, such as animal and cellular behaviors. For instance, Jin and Meng [9] introduced morphogenetic robotics as an interesting category of methodologies for designing self-organizing multirobotic systems, which are inspired by genetic and cellular mechanisms governing biological morphogenesis. Along this line of research, a hierarchical gene regulatory network (h-GRN) was proposed for multi-robot shape formation [10]. The top layer of the h-GRN receives positional information of neighboring robots and targets and generate enclosing patterns around the targets, while the bottom layer drives the robots onto the generated patterns in a self-organized way. However, this algorithm requires each robot to be able to access the position and orientation information of its neighbors and obstacles nearby. This assumption enables robots to estimate the position of the obstacles in a global coordinate system. With a similar approach, Peng et al. [11] presented a modified GRN to surround multiple diffusive targets with a predefined pattern. Unfortunately, this assumption can hardly hold for minimalist and miniaturized robots. In [12], a method for path planning with obstacle avoidance was presented for micro-robotic drug delivery systems, where it is assumed that the position of the targeted cells is known to the micro-robots. Kubo et al. [13] defined swarm based task allocation method to trap targets whose global positions are not available for individual robots.

There are other examples of designing methods for swarm robotic aggregation and shape formation. In [14], the robots are assumed to have multiple infrared (IR) sensors, while in [15] the robots need to use integrated scalable map. To the best of our knowledge, little research work has been reported in which neither position nor velocity is required [16], [17].

\section{Constraints and limitations in miniaturized swarm robotic systems}

It has ben becoming increasingly popular to manufacture micro-robotic systems consisting of microprocessors, micro-sensors, micro-actuators and microscale data exchange devices. In the field of micromedical robotics, it is hoped that, in a not very far future, thousands molecular-size robots are injected into blood vessels, or swallowed by patients to accurately tackle tumors, destroy cancer cells or clean arteries. 
Other applications of miniaturized robots can also be imagined, such as removal of pollutants and toxic microscale debris from water resources [18] and inspection of small structures.

Micro-scale robotic systems are typically highly noisy and difficult to control. Many capabilities in normal robots, such as global positioning, long-range communication, motion feedback, sensor arrays are no longer in micro-scale robots. For example, ultra-lightweight autonomous systems are easily impeded by colliding with each other or with obstacles in the environment. Typically, the signal-to-noise ratio in such systems is very low. Hence, a large amount of uncertainties in measurements and motion control are expected in these systems [19]. Due to the above reasons, enhancement of measurements and accuracy of motion control in microscales can be very costly. In addition, precise measuring and locomotion devices consume more energy. Therefore, limited power supply strongly restricts the number and capacity of devices that can be mounted on microrobots [20], [21].

Kilobots are a good example of robots with highly constrained and noisy sensing and motion capabilities. The communication range of Kilobots is just a few times of its size and heavily depends on the characteristics of the surface they are moving on. Each robot receives message packages composed of nine bytes broadcast two times per second. There is no accurate control over the motion speed of a Kilobot. Its locomotion mechanism is very simple and composed of two vibrating motors. The motors are off or vibrating at a certain frequency. To calibrate, each motor is adjusted to a fixed frequency, and consequently, the robots are not able to change their rotation speeds. It is non-trivial either for Kilobots to travel in a straight line. The sole feedback that they can rely on is the measurement of the distance to their neighbors. However, this distance is calculated based on the strength of the received IR signals from neighboring robots. The strength of these signals are affected by many factors, such as the body of the neighboring robots in the middle and surface they are moving on. The movement of Kilobots is also affected by collisions between the robots. They do not have any proximity sensor for detecting collisions with other robots. Hence, Kilobots can be seen as very typical micro-robotic systems.

\section{TARget MORPhOGEN DifFusion AND REGULATION}

Morphogens are chemical substances that diffuse through biological tissues and create concentration gradients. Morphogens can interact with each other as an activator or inhibitor and generate spatial patterns. These patterns can inform cells about their spatial location inside the tissue and their relevant tasks or behaviors, among others. Inspired by the morphogen mechanisms in developmental biology, we suggest in this work a method for forming a swarm aggregation evenly distributed around a target based on artificial morphogen gradients. We assume that morphogen gradients emitted by targets can be detected and interpreted by the robots in the neighborhood of the targets. These robots can also pass morphogen concentration information onto their neighboring robots and this process continues until the diffusion process stabilizes. We use the following equation to simulate morphogen diffusion:

$$
\frac{C^{i}}{d t}=\alpha \sum_{j}^{N^{i}}\left(\frac{R}{d^{i j}}\right) \cdot C_{j}-\gamma \cdot C^{i} \quad \begin{gathered}
\alpha, \gamma>0 \\
0<C^{i}<C_{M A X}
\end{gathered}
$$

where, $C^{i}$ represents the morphogen concentration of robot $i, C_{M A X}$ is the maximum concentration, $d^{i j}$ indicates the distance between robot $i$ and robot $j, R$ is the radius of each robot for the purpose of normalization and non-dimentionalization, $N^{i}$ is the number of neighbors of robot $i, \alpha$ and $\gamma$ symbolize the diffusion rate and decay rate, respectively. Fig. 2(a) illustrates the morphogen concentration in an aggregation with a morphogen source in the middle. The level of the morphogen concentration is the highest in the source location $\left(C_{M A X}\right)$. Fig. 2(b) shows the morphogen concentration of the robots in term of their distances to the source at different time instances starting from the beginning of diffusion. By choosing proper values for $\alpha$ and $\gamma$, a gradient of morphogen concentration can form from the source towards the boundary of the swarm aggregation, which reaches a steady state within a short period of time.

\section{A. Target Tracking}

In our previous work [22], we suggested a target tracking algorithm using morphogen diffusion for minimalist robots without directional sensing and self-localization. Based on the calculated morphogen concentration in the previous positions, a robot is able to decide in which direction and how much it should rotate in order to be aligned with the direction of the increasing or decreasing morphogen concentration. The direction of morphogen gradients can be estimated using a number of previous estimated positions in the robots coordinate system. A robot can estimate its position in its local coordinate system as follows:

$$
\mathbf{P}_{t+1}=\mathbf{P}_{t}+\hat{\boldsymbol{\theta}}_{t} V d t
$$

where $\mathbf{P}_{t}$ is the current position, $\mathbf{P}_{(t+1)}$ is the next position, $V$ is the velocity of the robot, and $\hat{\boldsymbol{\theta}}_{t}$ is a 


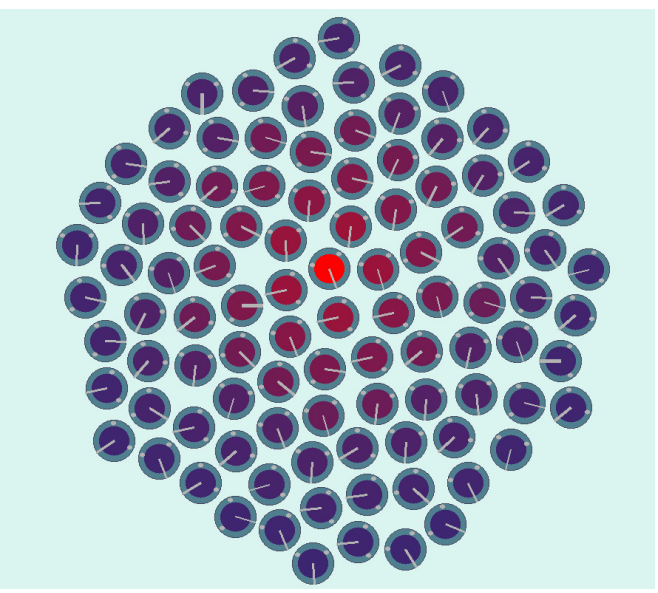

(a)

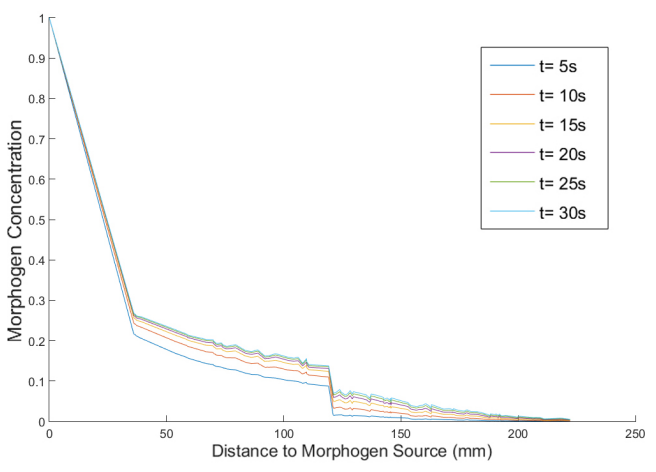

(b)

Fig. 2: Morphogen diffusion from a source in the middle of an aggregation. a) The intensity of red colour indicates the level of morphogen concentration. b) Morphogen concentration in term of distance to the source.

unit vector determining the direction of the movement, and $d t$ is the change of time. In this way, a robot can approximately self-localize its position. Note, however, that the error in estimating the position of the robot will accumulate over time due to inaccurate movements. The direction of the morphogen gradient can be estimated using the following equations:

$$
\begin{gathered}
\Delta \mathbf{C}_{t, i}=\frac{\mathbf{P}_{t, i}-\mathbf{P}_{t-1, i}}{\left\|\mathbf{P}_{t, i}-\mathbf{P}_{t-1, i}\right\|}\left(C_{\mathbf{P}_{t, i}}-C_{\mathbf{P}_{t-1, i}}\right), \\
\mathbf{C}_{T, i}=\sum_{T=t-N_{S}}^{t} \Delta \mathbf{C}_{T, i}, \\
\hat{\mathbf{C}}_{T, i}=\frac{\mathbf{C}_{T, i}}{\left\|\mathbf{C}_{T, i}\right\|},
\end{gathered}
$$

where $\mathbf{P}_{t, i}$ represents the position of robot $i$ at timestep $t, C_{\mathbf{P}_{t, i}}$ is the morphogen concentration measured at position $\mathbf{P}_{t, i}, N_{s}$ is the number of the previous time-steps taken into account to evaluate the morphogen gradient, $\|$.$\| returns the magnitude of a vector, and \hat{\mathbf{C}}_{T, i}$ is a unit vector indicating the orientation of the morphogen concentration.

At each iteration, a robot updates its current direction using Eq. (5):

$$
\begin{aligned}
& \Delta \boldsymbol{\theta}_{t}=\Delta \hat{\mathbf{C}}_{t}-\hat{\boldsymbol{\theta}}_{t-1}, \\
& \operatorname{sat}\left(\Delta \theta_{t}\right)=\left\{\begin{array}{l}
\left\|\Delta \boldsymbol{\theta}_{t}\right\|=\Delta \boldsymbol{\theta}_{\max }, \quad \text { if }\left\|\Delta \boldsymbol{\theta}_{t}\right\|>\Delta \theta_{\max } \\
\Delta \theta_{t}, \quad \text { otherwise }
\end{array}\right. \text {, } \\
& \hat{\boldsymbol{\theta}}_{t}=\hat{\boldsymbol{\theta}}_{t-1}+\operatorname{sat}\left(\Delta \theta_{t}\right)
\end{aligned}
$$

In experiments, we need to take limitations of the rotational speed into account, i.e., the change in direction will be bounded if the change in direction in one step of the simulation is greater than $\Delta \theta_{\max }$, referring to Eq. (7). Fig. 3 shows the snapshots of the path of approaching a target robot by a follower.

Here, we use the same mechanism for approaching a diffusive target. Target tracking only cannot guarantee a complete and robust enclosure of the target in all sides. As shown in Fig. 4, tracking and reaching a target may not lead to a reliable surrounding of the target when the distribution of the robots around the target is extremely uneven. However, an even surrounding of the target is non-trivial for robots with only limited local awareness. In the following sections, we will investigate how to take advantage of morphogen diffusion to guide robots to the parts of a swarm where insufficient robots are aggregated.

\section{B. Edge Morphogen Diffusion and Regulation}

Reaction and diffusion of morphogens in cellular organs can result in diverse and complex patterns [23], [24]. By adding the reaction term into Eq. (1), we get:

$$
\frac{\Delta C_{b i}}{\Delta t}=\sum_{j} w_{i j} f_{i j}\left(C_{b i}, C_{b j}\right)+D_{i} \sum_{b^{\prime}=1}^{N_{b}} \frac{\left(C_{b i}-C_{b^{\prime} i}\right)}{n_{i} d_{b b^{\prime}}}-r_{i} C_{b i}
$$

where $w_{i j}$ represents the interaction matrix and $f_{i j}$ is the interaction function, which is usually defined by a sigmoid function. Turing was the first who explained reaction-diffusion of two morphogens [25], where one morphogen serves as an activator, while the other inhibits the expression of activator. Turing's reaction-diffusion model has found many successsful applications in the field of distributed control of self-organizing systems [26]. Inspired by his model, we defined Eq. (1), where the morphogen from the diffusive target in the middle of the aggregation inhibits the production of the second morphogen in the edge of aggregation. To achieve a circular-shape aggregation around the target, we consider a morphogen, named edge morphogen, produced 


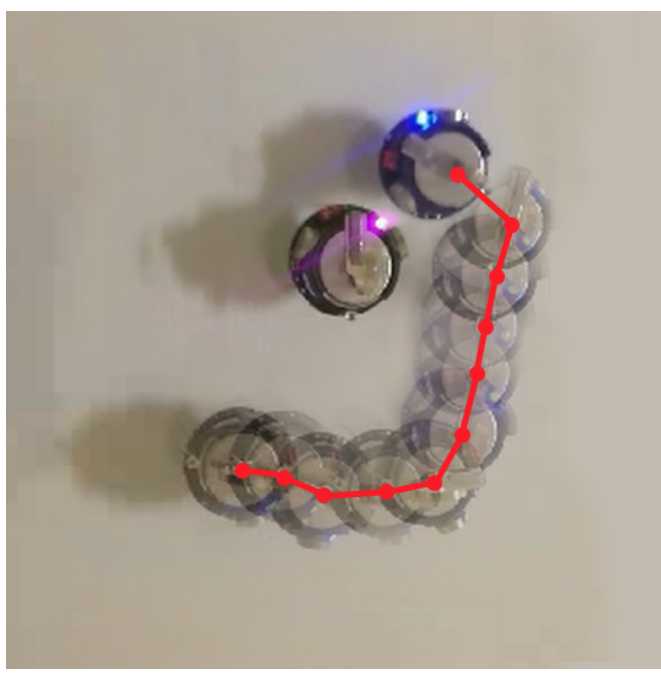

Fig. 3: Snapshot of approaching a morphogen source (denoted by the red LED bot) by a follower (blue LED bot).

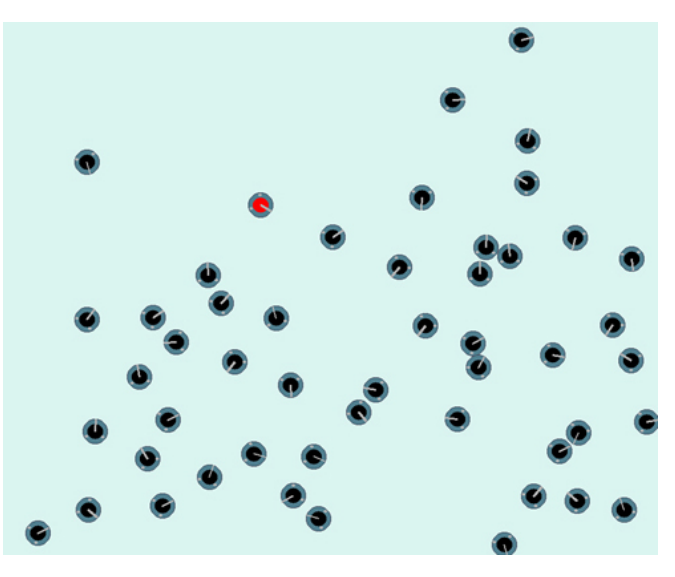

(a)

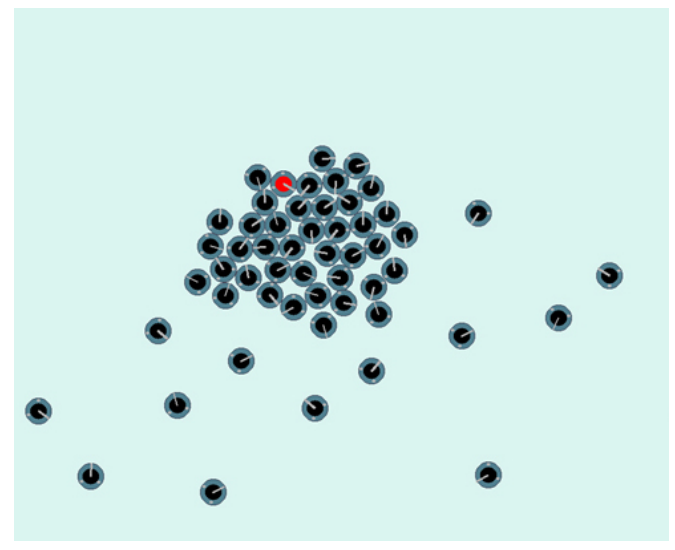

(b)

Fig. 4: Tracking a target does not always end up with fully surrounding the target. and diffused only by the robots at the boundary of the aggregation, which we call edge robots. We define the diffusion-reaction equation of the edge morphogen, which is regulated by the target morphogen:

$$
\begin{gathered}
\frac{d C_{E}^{i}}{d t}=\varphi \cdot \operatorname{sig}\left(C_{M A X}-C_{T}^{i}, k, m\right)-\omega \cdot \operatorname{sig}\left(C_{T}^{i}, k, m\right)+ \\
\alpha \sum_{j}^{N^{i}}\left(\frac{R}{d^{i j}}\right) \cdot\left(\frac{C_{E}^{j}-C_{E}^{i}}{C_{M A X}}\right) \cdot C_{E}^{j}-\gamma_{E} \cdot C_{E}^{i} \\
\operatorname{sig}(x, k, m)=\frac{2}{\pi} \operatorname{atan}(k \cdot(x+m))
\end{gathered}
$$

where, $C_{T}$ and $C_{E}$ represent the concentration of target morphogen and edge morphogen, respectively. From the above equation, we can observe that the production rate of the edge morphogen increases by decreasing of the target morphogen. Fig. 5 illustrates the result of diffusion and regulation of the edge morphogen for an irregularshape aggregation.

If a robot does not find another robot in its neighbourhood with a higher edge-morphogen, it will identify itself as an edge target point and set a certain bit in its message to inform the free robots (those have not yet joined the swarm). The free robots perform target-tracking until they join the aggregation around the targeted points. If a robot is close to the aggregation but there is no edge target point in its neighborhood, it starts to perform edge-following before reaching an edge target point. A few bits are allocated in the robots message packets for broadcasting the robots status so that the robots can be aware of their neighbors status. There is a dedicated bit in the robots' message packets to indicate if a robot is in 


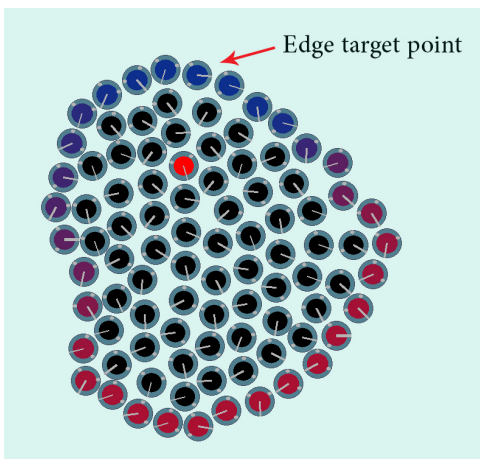

(a)

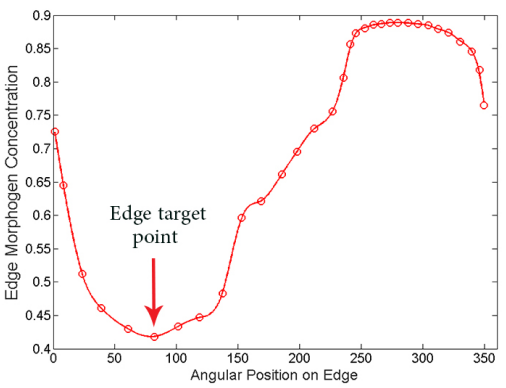

(b)

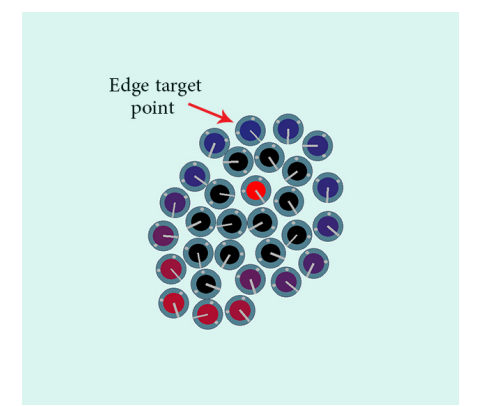

(c)

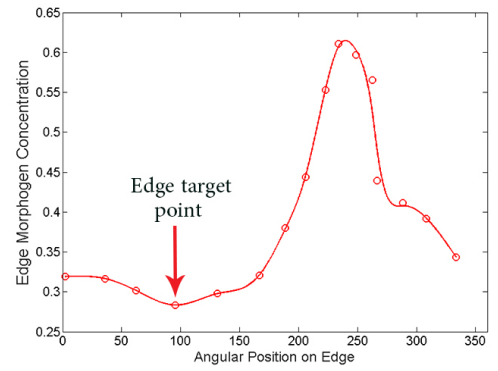

(d)

Fig. 5: Edge morphogen diffusion from a target at the middle of an aggregation indicated by red color a) Edge morphogen concentration for an aggregation of 100 robots. The edge robots' colors indicate the concentration of the edge morphogen, blue for low and red for higher concentration b) The curve represents the edge morphogen concentration of each robot in term of its angular position which is defined as the angle that it makes with the target. c) Edge morphogen concentration of edge robots for an aggregation of 30 robots, and d) is its associated edge morphogen concentration. the edge-following status. We explain in the following how it can be useful.

As collisions between the robots introduce lots of noise into robot's navigation, we implement a priority queue strategy for edge-following and target-tracking robots. To prevent collision between robots while they perform edge-following, a free robot switches to a waiting status if there is a robot in its neighborhood performing edge-following and has a higher target morphogen concentration. A robot in waiting status does not move to give priority to the robots that are closer to the edge to joint the aggregation first.

In a sequential manner, a robot stops moving if it has a robot in its neighbourhood in waiting status and closer to the swarm. It means that the first layer of waiting robots generate another layer of waiting robots. But this second layer do not changing their status into waiting to avoid having the whole swarm stop moving. In this way, there are two layers of the robots that stop moving and allow robots in edge following status joint the aggregation.

\section{Edge Differentiation Based on Morphogen Concen- tration}

In this algorithm, the robots at the boundary of the swarm should differentiate into edge robots to allow the production and diffusion of the edge morphogen for indicating where on the boundary a free robot should join. When an edge robot is covered by another layer of robots, it must differentiate back into a normal aggregated robot. In the literature, the number of neighbors or neighbors neighbors usually is utilized as a criterion for edge differentiation [22], [27]. If this number falls below a certain threshold, a robot will differentiate into an edge robot. However, this threshold depends on the swarm size. Moreover, for a robot swarm having a non-convex shape, the above method may fail to work properly. Therefore, we cannot simply rely on the number of neighbors or neighbors neighbors for determining the points where free robots should join as the size of the enclosure swarm grows, or when the shape is non-convex at some points of growth.

Using the target morphogen concentration of robots' neighbors, we suggest an edge differentiation criterion that is invariant to the size and convexity of the shape of the swarm, and the location of the target inside the swarm as well. In our method, a robot counts the number of neighbors with a low morphogen concentration within a certain distance. If this number is below a predefined threshold, the robot differentiates into an edge robot. Once the number is larger than the threshold again, the robot is no longer an edge robot. Fig. 6 shows 
the simulation results of edge differentiation for swarms of different sizes, shapes and target locations. We can see from the figure that the simulated Kilobots identify themselves as edge-robots if there are four robots within seven centimeters with a lower target morphogen concentration.

\section{RESULTS}

\section{A. Single target enclosure}

We conducted a simulation with 100 simulated Kilobots, which are scattered unevenly over a confined area with the dimensions of 120 by 120 centimeters. Fig. 7 depicts the snapshots of the process of surrounding a single target. The results show that the algorithm is able to regulate the circular shape of the aggregation around the target.

According to Fig. 5, when the shape of a robot aggregation is very different from an ideal disc, there is only one minimum through the edge. This is because a big difference in the morphogen rate between the edge robots creates a steep gradient. The closer the shape of the swarm to an ideal circle, the more smooth the edge morphogen through the edge layer will be. Thus, there might be multiple points of attraction on the boundary when the shape is round. Actually, this is useful since it can speed up the enclosure when the swarm aggregation is close to a circle. Fig. 8 presents the edge morphogen concentration of the edge robots (Fig. 8(a)) and their distances to the target (Fig. 8(b)) during an enclosure process. As can be seen in Fig. 8, the shape adaptation at the beginning and final stages of the enclosure is smoother than that in the middle stage. Note that the production and diffusion of morphogen do not happen immediately after a free robot joins the swarm. Once a free robot joins the robot swarm at a point of attraction, it takes time before the edge morphogen of the attractive robot (the edge robots on the points of attraction) exceeds that of its neighbors. Therefore, a point of attraction remains its status within a short period of time after a free robot is joined. As a result, additional robots may join the swarm on that point. Thus, fluctuations may occur in the edge morphogen gradient if the rate of free robots joining the robot aggregation is too high compared to the speed of morphogen diffusion and regulation. However, we are not allowed to increase the diffusion rate, since the morphogen gradient will quickly become smooth and thus lose its sensitivity to the shape of robot aggregation.

\section{B. Multiple Target}

Fig. 9 shows the result of a multiple target enclosure, where the targets are too far away to be surrounded by a single swarm. The enclosure of the targets might end up with different sizes either due to different initializations of the free robots, or to different locations of the targets in the field. However, when the targets are close enough to be surrounded by a single swarm, the final shape of the aggregation ends up with a single circular shape (Fig. 10). The diffusion of the edge morphogen through the boundary of all robot swarms regulates their growth. Based on this, predefined shapes can emerge through the initialization of the targets. Fig. 11 shows the final stages of swarms with different initializations of the targets.

\section{Experiment using Kilobots}

We conducted an experiment using 50 Kilobots for enclosure of one target. The experimental results are shown in Fig. 12. In the first run, we uploaded the simulated program in Kilobot without any changes. The robots are initialized by scattering unevenly over the field. The field itself is confined by barriers for Kilobots. However, Kilobots easily get stuck on the boundaries, especially in the corners due to their limited mobility. In this case, we need to rotate the stuck robots on the boundaries to let them move back into the field. Hence, the barriers are not covered in the photos and footages. Fig. 12(a) shows the snapshot of the enclosure procedure. From the figure, we can conclude that the proposed algorithm works properly since the robot swarm successfully surrounds the target with a fairly evenly distributed circular shape.

For comparison, we removed the shape adaptation procedure from the enclosure algorithm and conducted the experiment again. The results, as depicted in Fig. 12(b), show that the final shape of aggregation is irregular and the enclosure around the target is very irregular.

\section{CONCLUSION AND FUtURE WORKS}

In this work, an algorithm for simulating morphogen diffusion and regulation was proposed to self-organize minimalistic robots for target surrounding. We explore how simulated diffusion and regulation of morphogens can be used to self-organize robots to form a evenly distributed swarm shape. We showed that the proposed mechanism can result in an adaptive growth of the robot aggregation. The algorithm was successfully implemented in both simulations and physical experiments. It was demonstrated that with the proposed adaptation mechanism is able to guide the robots to form a more evenly distributed aggregation surrounding the targets. In addition, we showed that the proposed algorithm is able to automatically surround multiple targets with a single swarm or multiple swarms, depending on whether the targets far away or close by. We also revealed that a 

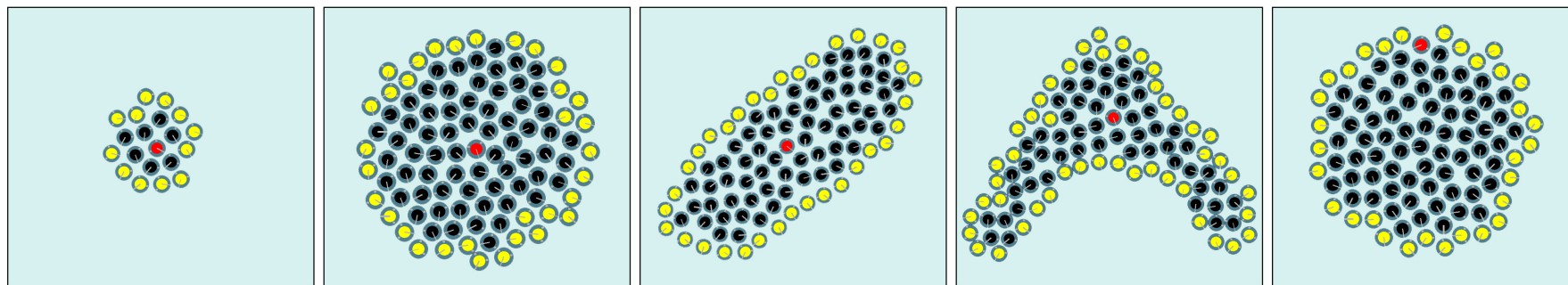

Fig. 6: Edge differentiation for robot aggregations with different sizes, shapes and target locations. The red circle indicates the target, and yellow ones are the agents differentiated to edge.
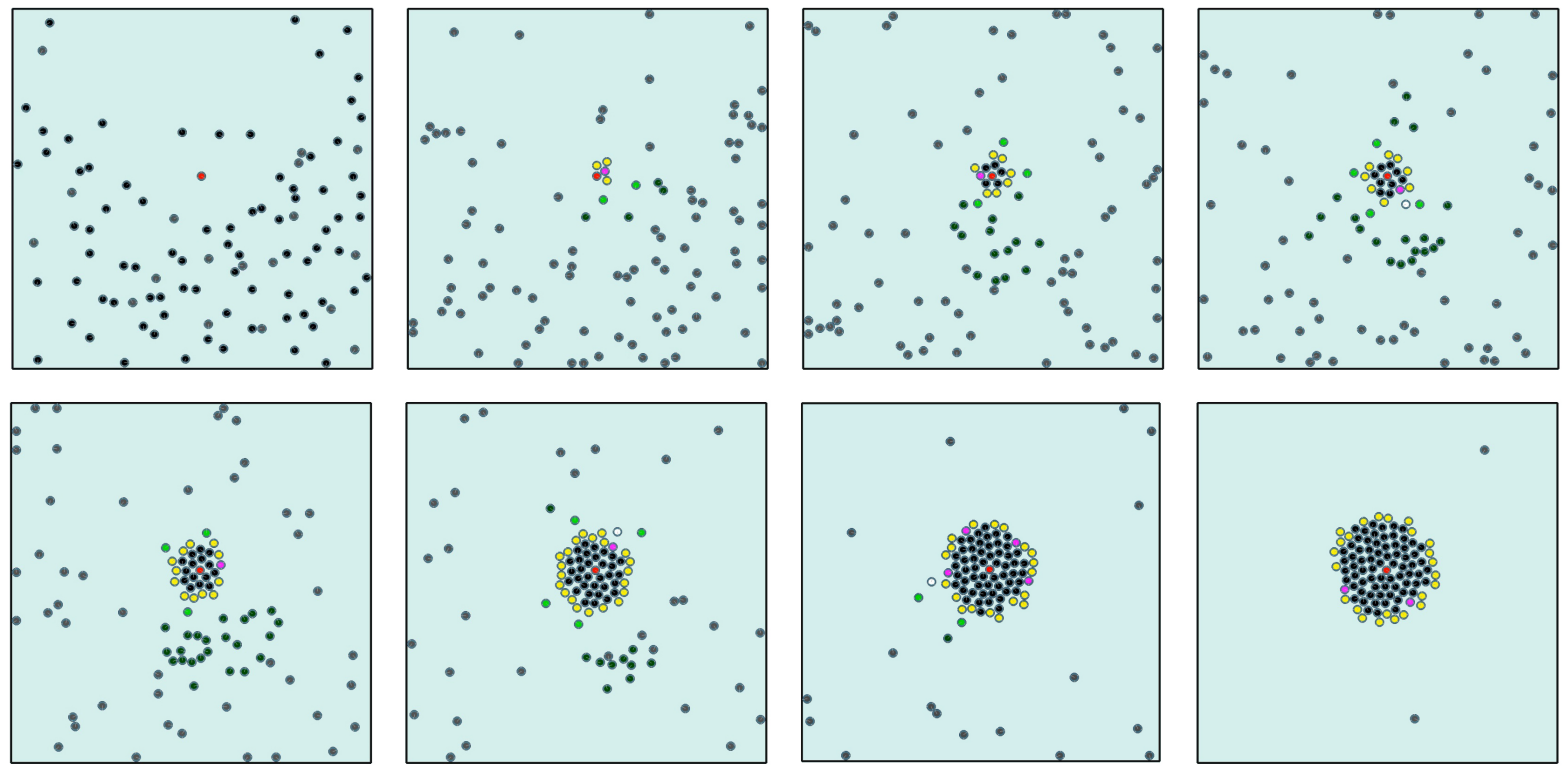

Fig. 7: Target enclosure over time by 100 robots. Time increases from up-left to bottom-right. The robots show yellow colour once they differentiate into an edge robot, pink color for being edge target, green while doing edge-following, and white for direct target-tracking.

predefined shape can emerge by properly initializing the location of the targets.

Future work can include the exploration of proposed mechanism for forming more complex shapes in a selforganizing way with the help of morphogen regulation at the boundary of a robot aggregation. Furthermore, we can also consider moving robots away from the aggregation in adapting the swarm shape. It is expected that with a leaving mechanism and more morphogens, more advanced algorithms can be developed for shape adaptation for minimalistic robotic swarm.

\section{ACKNOWLEDGEMENTS}

This research is funded by the European Commission 7th Framework Program, Project No. 601062, SWARMORGAN.

\section{REFERENCES}

[1] J. M. Soares, A. P. Aguiar, A. M. Pascoal, and A. Martinoli, "A distributed formation-based odor source localization algorithm-design, implementation, and wind tunnel evaluation," in Robotics and Automation (ICRA), 2015 IEEE International Conference on. IEEE, 2015, pp. 1830-1836.

[2] A. Murai, H. Matsukura, R. Takemura, and H. Ishida, "Active airflow generation to assist robotic gas source localization: Initial experiments in outdoor environment," ECS Transactions, vol. 75, no. 16, pp. 65-72, 2016.

[3] J. Cao, M. Li, H. Wang, L. Huang, and Y. Zhao, "An improved bacterial foraging algorithm with cooperative learning for eradicating cancer cells using nanorobots," in Robotics and Biomimetics (ROBIO), 2016 IEEE International Conference on. IEEE, 2016, pp. 1141-1146.

[4] T. A. Witten and L. M. Sander, "Diffusion-limited aggregation," Physical Review B, vol. 27, no. 9, p. 5686, 1983.

[5] V. Crespi, A. Galstyan, and K. Lerman, "Top-down vs bottomup methodologies in multi-agent system design," Autonomous Robots, vol. 24, no. 3, pp. 303-313, 2008.

[6] V. Trianni, Evolutionary swarm robotics: evolving self- 


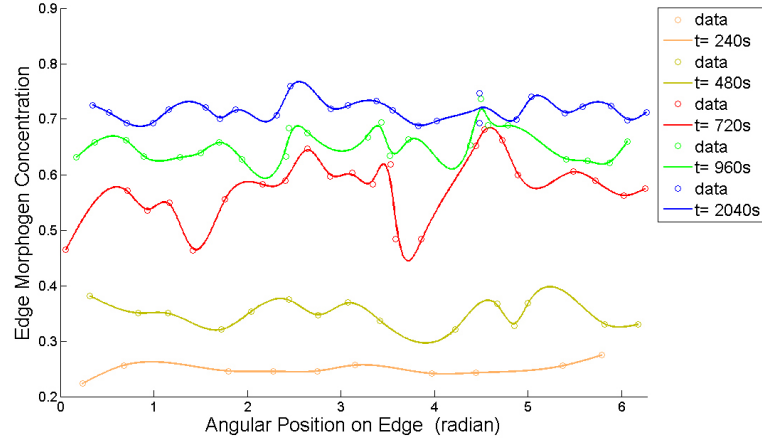

(a)

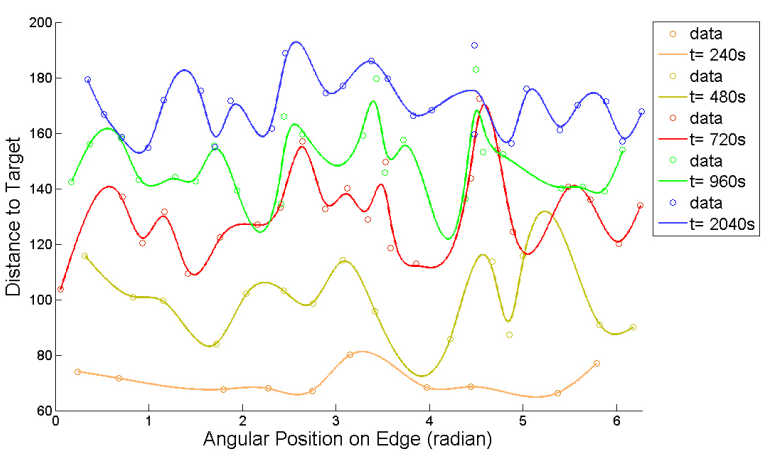

(b)

Fig. 8: Growth adaptation of the robot aggregation. a) The edge morphogen concentration of the edge robots increases as the aggregation grows. b) The distance from the edge robots to the target.

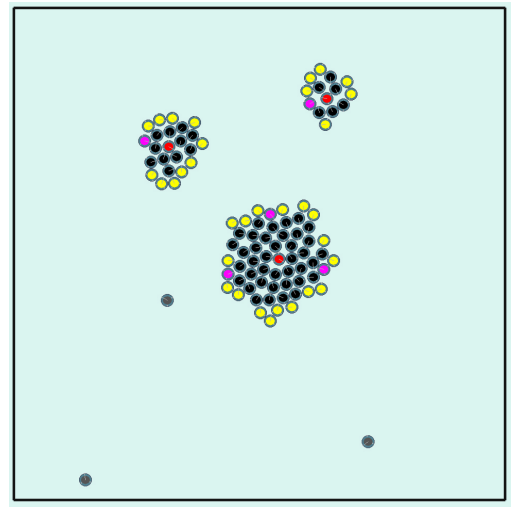

Fig. 9: Multi-target enclosure with three separated targets. If the edges of robot swarms do not overlap, the swarm may end up with different sizes. organising behaviours in groups of autonomous robots. Springer, 2008, vol. 108.

[7] A. Brutschy, G. Pini, C. Pinciroli, M. Birattari, and M. Dorigo, "Self-organized task allocation to sequentially interdependent tasks in swarm robotics," Autonomous agents and multi-agent systems, vol. 28, no. 1, pp. 101-125, 2014.

[8] M. Brambilla, E. Ferrante, M. Birattari, and M. Dorigo, "Swarm robotics: a review from the swarm engineering perspective," Swarm Intelligence, vol. 7, no. 1, pp. 1-41, 2013.

[9] Y. Jin and Y. Meng, "Morphogenetic robotics: An emerging new field in developmental robotics," IEEE Transactions on Systems, Man, and Cybernetics, Part C: Applications and Reviews, vol. 41, no. 2, pp. 145-160, 2011.

[10] Y. Jin, H. Guo, and Y. Meng, "A hierarchical gene regulatory network for adaptive multirobot pattern formation," IEEE Transactions on Systems, Man, and Cybernetics, Part B: Cybernetics, vol. 42, no. 3, pp. 805-816, 2012.

[11] X. Peng, S. Zhang, and X. Lei, "Multi-target trapping in constrained environments using gene regulatory network-based pattern formation," International Journal of Advanced Robotic Systems, vol. 13, no. 5, p. 1729881416670152, 2016.

[12] A. Banharnsakun, T. Achalakul, and R. C. Batra, "Target finding and obstacle avoidance algorithm for microrobot swarms," in Systems, Man, and Cybernetics (SMC), 2012 IEEE International Conference on. IEEE, 2012, pp. 1610-1615.

[13] M. Kubo, H. Sato, A. Yamaguchi, and T. Yoshimura, "Target enclosure for multiple targets," Intelligent Autonomous Systems 12, pp. 795-803, 2013.

[14] O. Soysal and E. Sahin, "Probabilistic aggregation strategies in swarm robotic systems," in Swarm Intelligence Symposium, 2005. SIS 2005. Proceedings 2005 IEEE. IEEE, 2005, pp. 325-332.

[15] A. scalable size selection for the shape of a distributed robotic collective, "Automatic scalable size selection for the shape of a distributed robotic collective," in Intelligent Robots and Systems (IROS), 2010 IEEE/RSJ International Conference on. IEEE, 2010, pp. 508-513.

[16] C. Cheah, S. Hou, and J. Slotine, "Region-based shape control for a swarm of robots," Automatica, vol. 45, pp. 2406-2411, 2009.

[17] X. Yan, J. Chen, and D. Sun, "Multilevel-based topology design and shape control of robot swarms," Automatica, vol. 48, no. 12, pp. 3122-3127, 2012.

[18] G. M. Patel, G. C. Patel, R. B. Patel, J. K. Patel, and M. Patel, "Nanorobot: a versatile tool in nanomedicine," Journal of Drug Targeting, vol. 14, no. 2, pp. 63-67, 2006.

[19] R. J. Wood, "The challenge of manufacturing between macro and micro," American Scientist, vol. 102, no. 2, p. 124, 2014.

[20] C. Huang, J.-a. Lv, X. Tian, Y. Wang, Y. Yu, and J. Liu, "Miniaturized swimming soft robot with complex movement actuated and controlled by remote light signals," Scientific reports, vol. 5, 2015.

[21] S. S. Baek, "Autonomous ornithopter flight with sensor-based behavior," Univ. California, Berkeley, Tech. Rep. UCB/EECS2011-65, 2011.

[22] A. Ramezan Shirazi, H. Oh, and Y. Jin, "Morphogenetic selforganization of collective movement without directional sensing," in Advances in Autonomous Robotics Systems. Springer, 2014, pp. 139-150.

[23] H. Meinhardt and A. Gierer, "Pattern formation by local selfactivation and lateral inhibition," BioEssays, vol. 22, pp. 753760, 2000.

[24] J. Davies, Mechanisms of Morphogenesis. Elsevier Academic Press, 2005

[25] A. Turing, "The chemical basis of morphogenesis," Philosoph- 

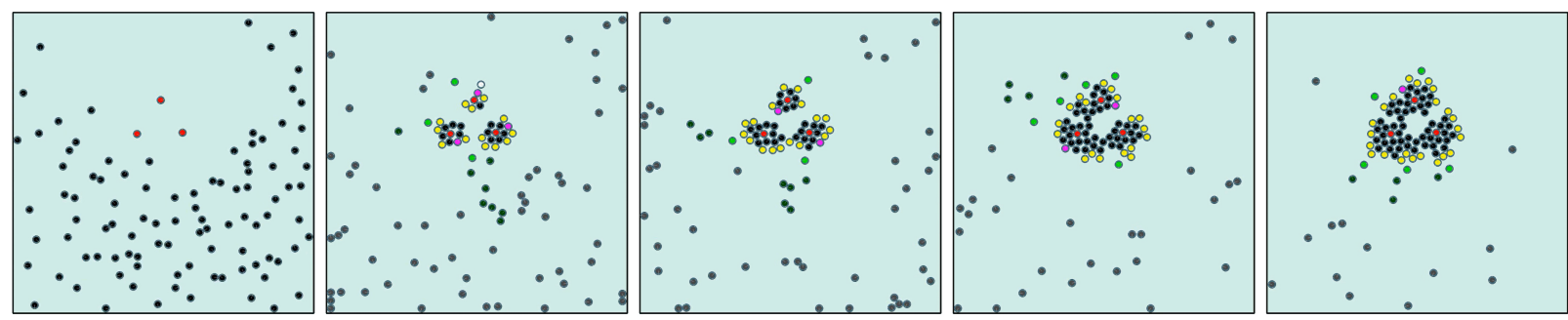

Fig. 10: Enclosure of three closely located targets. When the edge layers overlap, the diffusion of the edge morphogen regulates the shape of the robot aggregation.
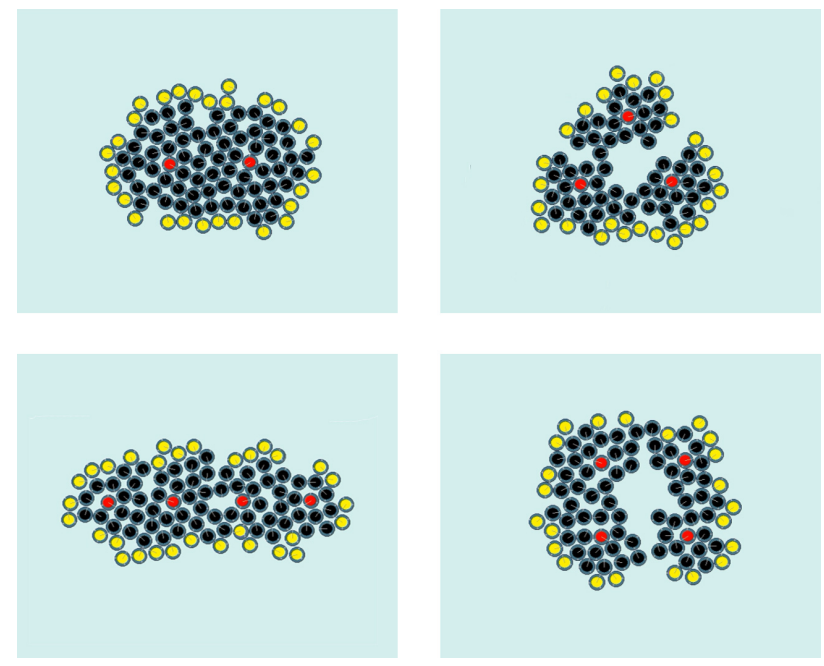

Fig. 11: The formation of predefined shapes based on the initial locations of the targets.

ical Transactions of the Royal Society B, vol. 237, pp. 37-72, 1952.

[26] H. Oh, A. R. Shirazi, C. Sun, and Y. Jin, "Bio-inspired selforganising multi-robot pattern formation: A review," Robotics and Autonomous Systems, vol. 91, pp. 83-100, 2017.

[27] C. S. Sahin, E. Urrea, and M. U. Uyar, "Bio-inspired techniques for topology control of mobile nodes," Formal and Practical Aspects of Autonomic Computing and Networking: Specification, Development, and Verification, pp. 217-240, 2011.

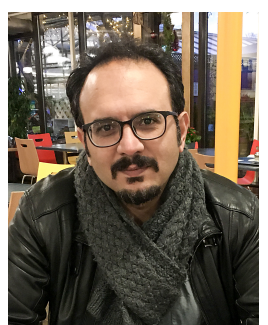

Ataollah Ramezan Shirazi received the BS degree in Solid State Physics from University of Tehran, Tehran Iran, in 2008, the MS degree in Mechatronics Engineering from University of Tabriz, Tabriz, Iran, in 2010, and the $\mathrm{PhD}$ degree in bio-inspired self-organizing algorithms from the Department of Computer Science from University of Surrey, Guildford, UK in 2017.

Since 2017, he is a researcher in Adaptive Systems Research Group at the University of Hertfordshire, Hatfield, UK, where he has been working on multidisciplinary research topics such as informationtheoretic modelling and analysis of sensorimotor contingencies in robot-robot interactions, and designing and development of a multiagent agricultural robotic platform. His research interests cover cognitive architecture for multi-agent robotic systems, self-organization and collective behaviour, self-adaptation, computational intelligence and cognitive science. 

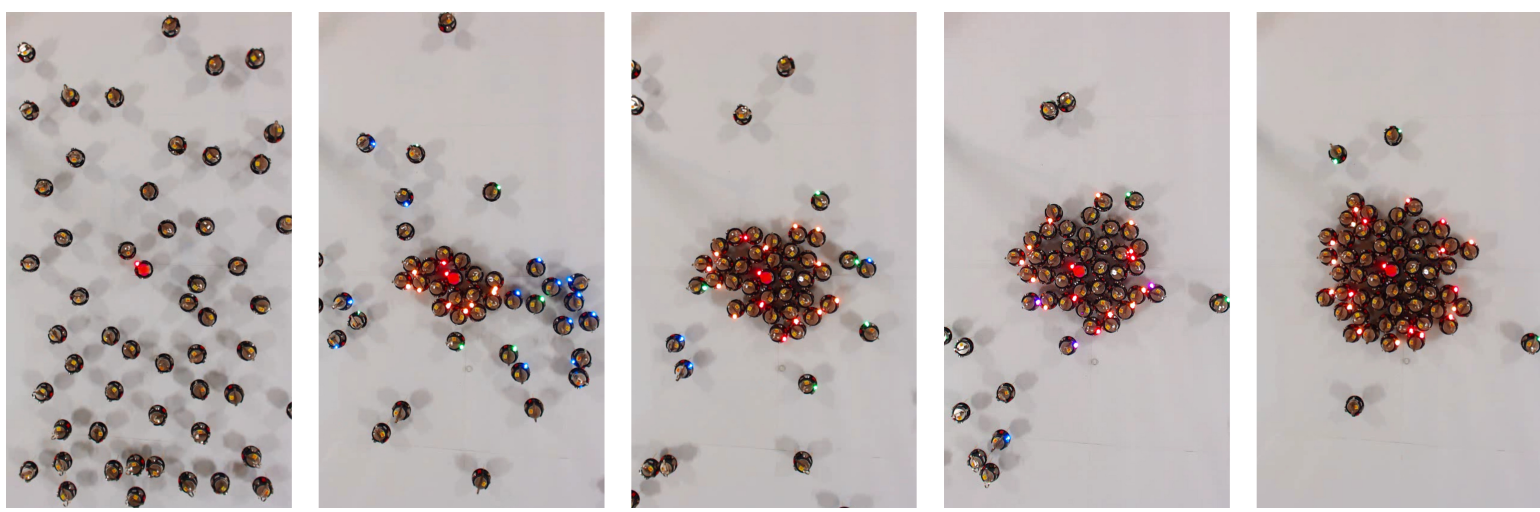

(a)
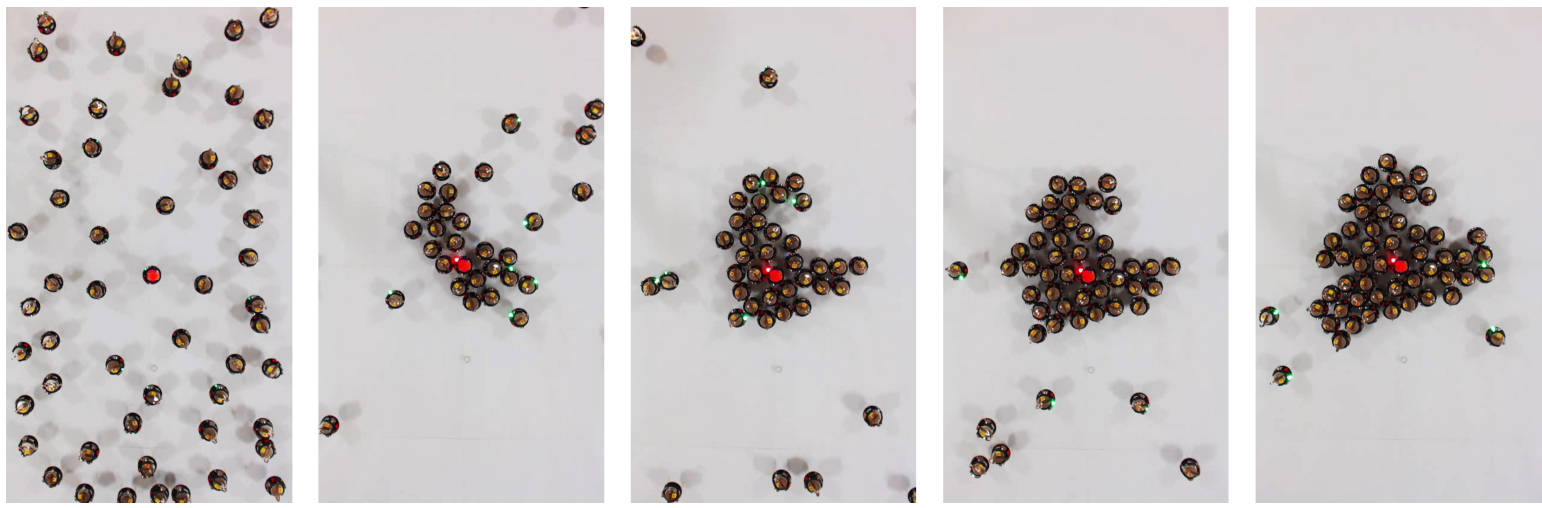

(b)

Fig. 12: Growth adaptation of the robot aggregation. a) The edge morphogen concentration of the edge robots increases as the aggregation grows. b) Distance from the edge robots to the target.

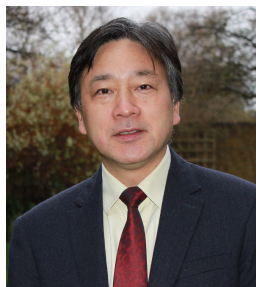

Yaochu Jin (M'98-SM'02-F'16) received the B.Sc., M.Sc., and Ph.D. degrees from Zhejiang University, Hangzhou, China, in 1988, 1991, and 1996, respectively, and the Dr.-Ing. degree from Ruhr University Bochum, Germany, in 2001.

$\mathrm{He}$ is a Distinguished Chair, Professor in Computational Intelligence with the Department of Computer Science, University of Surrey, Guildford, U.K., where he heads the Nature Inspired Computing and Engineering Group. He was also a Finland Distinguished Professor funded by the Finnish Funding Agency for Innovation (Tekes) and a Changjiang Distinguished Visiting Professor appointed by the Ministry of Education, China. He has (co)-authored over 300 peer-reviewed journal and conference papers and been granted eight patents on evolutionary optimization. He has delivered 30 invited keynote speeches at international conferences. His main research interests include data-driven surrogate-assisted evolutionary optimization, multi-objective evolutionary learning, privacy-preserving and adversarial machine learning, and evolutionary developmental systems.

Dr. Jin is the recipient of the Best Paper Award of the 2010 IEEE Symposium on Computational Intelligence in Bioinformatics and Computational Biology, the 2015 and 2017 IEEE Computational Intelligence Magazine Outstanding Paper Award, and the 2018 IEEE Transactions on Evolutionary Computation Outstanding Paper Award. $\mathrm{He}$ is the Editor-in-Chief of the IEEE Transactions on Cognitive and Developmental Systems and the Co-Editor-in-Chief of Complex \& Intelligent Systems. He was an IEEE Distinguished Lecturer, and
Vice President for the Technical Activities of the IEEE Computational Intelligence Society from 2014 to 2015 . He was named "a Highly Cited Researcher for 2019" by the Web of Science group. He is a Fellow of IEEE. 